\title{
Unknown cause of non-PTH mediated hypercalcemia in Pregnancy
}

Authors: Raju Panta, Anotine Makdissi, Manav Batra, Ajay Chaudhuri

Hospital: University at Buffalo, Buffalo, New York, USA

\section{Introduction}

Calcium metabolism during pregnancy changes significantly but ionized calcium level remains normal.

During pregnancy, there is 2-fold increase in intestinal calcium absorption mediated by increase in 1,25-dihydroxyvitamin $\mathrm{D}(1)$. Hypercalcemia in pregnancy is uncommon and most reported cases are primary hyperparathyroidism.

A 18-year-old 10 weeks' pregnant African American female presented to hospital with epigastric pain, nausea, vomiting for 2 days. She was not taking any calcium supplements. She had mild epigastric tenderness.

Lab showed hypercalcemia with corrected calcium of $13.1 \mathrm{mg} / \mathrm{dl}$ (normal 9-10.8) and ionized calcium of $6.7 \mathrm{mg} / \mathrm{dl}$ (normal 4.7-5.3). PTH was undetectable. PTHrP, thyroid function test, serum immunoelectrophoresis, vitamin A, ACE were normal. 25-hydroxyvitamin D was low, 9 (normal $30-100 \mathrm{ng} / \mathrm{ml}$ ) and 1,25-dihydroxyvitamin D was inappropriately normal 51 (Normal 19.9-79.3 pg/ml).

Her 24-hour urinary calcium was 436.8mg (normal 50-150). MRI of neck, chest, abdomen, pelvis showed a $1.4 \mathrm{~cm}$ sized lymph node in right hilar region. Foetal ultrasound revealed singleton pregnancy, clubbed left foot. Due to persistent hypercalcemia, she had PICC placed and discharged with iv hydration.

Lab from first pregnancy (medically terminated) two years ago showed corrected calcium of 13.5. Her calcium in between these pregnancies was 9.9. With intravenous hydration at home, her ionized calcium was between 5.5 to 6.9

Our case represents a unique case of non-PTH mediated hypercalcemia.

Extensive work up did not reveal any definite aetiology. Another consideration is CYP24A1 gene mutation. As CYP24A1 metabolizes 1,25-dihydroxyvitamin D and 25-hydroxyvitamin D, patients have high levels of both and kidney stones(2). Our patient did not have high levels of 25-hydroxyvitamin D and no renal stones. Normally, PTHrP level steadily rises throughout the pregnancy(1). Our patient had normal levels of PTHrP. A normal calcium level in between pregnancies suggest that this hypercalcemia is related to pregnancy.

Hence, we suspect there is/are unknown calcium regulators. 